\title{
Níveis nutricionais de cálcio para aves de corte ISA Label criadas sob semiconfinamento
}

[Dietary calcium levels for ISA Label broilers raised in semi-confined system]

\author{
S.R.F. Pinheiro ${ }^{1,5}$, N.K. Sakomura ${ }^{2}$, J.C. Siqueira ${ }^{1}$, S.M. Marcato $^{1}$, L.R.B. Dourado ${ }^{1}$, \\ J.B.K. Fernandes ${ }^{3}$, E.B. Malheiros ${ }^{4}$ \\ ${ }^{1}$ Aluno de pós-graduação - FCAV-UNESP - Jaboticabal, SP \\ ${ }^{2}$ Departamento de Zootecnia - FCAV-UNESP - Jaboticabal, SP \\ ${ }^{3}$ CAUNESP - Jaboticabal, SP \\ ${ }^{4}$ Departamento de Ciências Exatas - FCAV-UNESP - Jaboticabal, SP \\ ${ }^{5}$ Bolsista do $\mathrm{CNPq}$ \\ RESUMO
}

\begin{abstract}
Determinaram-se os níveis nutricionais de cálcio (Ca) para aves, machos e fêmeas, da linhagem ISA Label, nas fases inicial (um a 28 dias), crescimento (28 a 56 dias) e final (56 a 84 dias). Foram realizados três ensaios, um para cada fase, e, em cada ensaio, 480 aves com idade correspondente à fase de criação foram alojadas em 24 unidades experimentais com áreas de abrigo e de pastejo. Foi utilizado delineamento inteiramente ao acaso, em esquema fatorial 4x2 (Ca e sexo), totalizando oito tratamentos com três repetições de 20 aves. Avaliaram-se: ganho de peso (GP); consumo de dieta (CD); conversão alimentar (CA); teores de fósforo (PT), de cálcio (CaT) e de cinzas na tíbia (CT) e resistência à quebra óssea (RQO). Na fase inicial, recomenda-se $1,16 \%$ de Ca na dieta, para aves de ambos os sexos, na fase de crescimento, 0,78 e 0,88\% de Ca para machos e fêmeas, respectivamente, e, na fase final, 0,69\% de Ca na dieta para ambos os sexos.
\end{abstract}

Palavras-chave: aves de corte, crescimento lento, fósforo, requisito nutricional

\begin{abstract}
The nutritional calcium (Ca) levels were determined for ISA Label broilers in the starter (1 to 28 days), growing (28 to 56 days), and finishing (56 to 84 days) phases. Three trials were conducted, one for each phase and in each trial, 480 birds with age corresponding to the phase were housed in 24 experimental units with shelter and pasture areas. The experimental design was completely randomized in a factorial arrangement of $4 \times 2$ (Ca levels and sex), totaling eight treatments with three replicates of 20 birds. Body weight gain (BWG); feed intake (FI); feed per gain (FG); contents of phosphorus (TP), calcium (TCa) and ash (TA) in tibia; and bone breaking strength (BSB) were evaluated. At the starter phase, $1.16 \%$ of $\mathrm{Ca}$ is recommended in the diet for birds of both sexes. At the growing phase, 0.78 and $0.88 \%$ of Ca for males and females, respectively, are recommended and at the finishing phase, the level of $0.69 \%$ of Ca is recommended in diet for both sexes.
\end{abstract}

Keywords: broilers, phosphorus, slow-growing birds, nutritional requirement

\section{INTRODUÇÃO}

Para pequenos e médios produtores rurais que visam aumentar sua renda, a criação de aves alternativas desponta-se como uma excelente oportunidade de negócios, pois, nos últimos

Recebido em 28 de agosto de 2009

Aceito em 22 de dezembro de 2010

E-mail: sandrazoot@yahoo.com.br anos, uma parcela crescente do mercado consumidor tem se interessado por produtos destas aves.

O fornecimento de dietas balanceadas às aves favorece seu desempenho produtivo. O cálcio e o fósforo são responsáveis por diversas funções 
orgânicas, mas a principal função é a mineralização da matriz óssea. Além da formação e manutenção da estrutura óssea, o cálcio é necessário para adequado crescimento, formação da casca do ovo, transmissão de impulsos nervosos, coagulação sanguínea, contração muscular, ativação de sistemas enzimáticos e envolvimento com a secreção de diferentes hormônios (Underwood e Suttle, 1999; Sá et al., 2004b).

A deposição de cálcio no esqueleto é mais intensa na fase de crescimento. Dessa forma, o conteúdo de cálcio no organismo dos pintinhos aumenta de maneira rápida na fase inicial, chegando, ao final de 30 dias de idade, a $80 \%$ do total de cálcio da ave adulta. Logo, inadequado fornecimento desse mineral durante a fase de crescimento terá como consequência um prejuízo no desenvolvimento da ave (Alves et al., 2002).

Tendo em vista a participação do Ca na estrutura óssea, a resistência à quebra óssea é uma variável importante para determinar a exigência desse mineral. Sá et al. (2004a) indicaram os níveis de 1,28 e 1,18\% de Ca para frangos de corte nas fases de crescimento e terminação, respectivamente, para essa variável. Em outro trabalho, Sá et al. (2004b) determinaram a exigência nutricional de $\mathrm{Ca}$ e sua biodisponibilidade em alguns alimentos para frangos de corte na fase de um a 21 dias, recomendando o nível de 1,20\% de Ca, com base nas respostas de resistência à quebra óssea.

Pesquisas que investigam as exigências de cálcio para aves de linhagens de crescimento lento são escassas, ou mesmo inexistentes, na literatura. Dessa forma, evidencia-se a necessidade de estudos visando avaliar os níveis nutricionais de cálcio para essas linhagens, visto que apresentam características de crescimento diferenciadas dos frangos de corte industriais. O objetivo deste trabalho foi determinar os níveis nutricionais de cálcio para aves da linhagem ISA Label nas fases inicial (um a 28 dias), crescimento (28 a 56 dias) e final (56 a 84 dias de idade), a fim de se elaborar programas de alimentação específicos para as linhagens de crescimento lento.

\section{MATERIAL E MÉTODOS}

Foram realizados três ensaios no período de 29 de janeiro a 23 de abril de 2007, para determinar as exigências de cálcio para aves da linhagem ISA Label de ambos os sexos, criadas em semiconfinamento nas fases inicial (um a 28 dias), crescimento (28 a 56 dias) e final (56 a 84 dias). Em cada ensaio, foram utilizadas 480 aves (240 machos e 240 fêmeas), distribuídas no delineamento experimental inteiramente ao acaso, com oito tratamentos, em esquema fatorial 4x2 (níveis de cálcio e sexo) e três repetições de 20 aves. Um lote de aves reservas para serem utilizadas nas fases de crescimento e final foi criado simultaneamente em galpão convencional até o $28^{\circ}$ e o $56^{\circ}$ dia.

As instalações experimentais consistiram de 24 piquetes, cada um dispondo de um abrigo $\left(3,13 \mathrm{~m}^{2}\right)$ e área de pastejo $\left(72,87 \mathrm{~m}^{2}\right)$. Os abrigos, com laterais teladas, providas de cortinas, cobertura de telha de cimento amianto e piso forrado com cama de maravalha, foram equipados com um comedouro tubular e um bebedouro tipo pendular. A área de pastejo, cercada por tela galvanizada, possuía predominantemente gramíneas do gênero Paspalum. Na fase inicial, as aves alojadas com um dia de idade permaneceram limitadas ao abrigo até o $28^{\circ}$ dia. Nas fases subsequentes, com acesso à área de pastejo, as aves eram soltas, diariamente, às $8 \mathrm{~h}$, e recolhidas ao abrigo às $18 \mathrm{~h}$.

Para cada fase, foram formuladas dietas basais (Tab. 1), compostas principalmente por milho e farelo de soja, considerando-se as exigências nutricionais de energia metabolizável, de aminoácidos (lisina e metionina + cistina) e de fósforo disponível de acordo com recomendações de Mendonça et al. (2008), Nascimento et al. (2009) e Pinheiro (2009), respectivamente. Os outros aminoácidos seguiram as relações aminoacídicas preconizadas por Rostagno et al. (2005). As dietas basais foram suplementadas com calcário em substituição ao inerte, resultando em dietas isonutritivas contendo 0,60; 0,82; 1,04 e 1,26\% de Ca na fase inicial; 0,49; 0,68; 0,87 e 1,06\% de Ca na fase de crescimento e 0,35; 0,54; 0,73 e 0,92\% de Ca na fase final. 
As aves receberam dieta e água à vontade e foram criadas somente com luz natural. O cálcio da forragem não foi considerado, pois, em criações em que as aves recebem dieta à vontade, o consumo de forragem é desprezível.

As variáveis avaliadas foram: ganho de peso (g/ave); consumo de dieta (g/ave); conversão alimentar (g de dieta consumida/g de ganho de peso); teores de cálcio (\%), de fósforo (\%) e de cinzas (\%) na tíbia; e resistência à quebra óssea (kgf/mm). Para avaliação dos parâmetros dos ossos, no final de cada ensaio, três aves de cada parcela, com peso corporal próximo ao da média da parcela ( $\pm 10 \%)$, foram submetidas a jejum alimentar de 12 horas e abatidas por deslocamento cervical para retirada das tíbias. As tíbias foram descarnadas e fervidas em água destilada por cinco minutos para facilitar a retirada dos resíduos de carne. A fíbula e as cartilagens também foram removidas.

Tabela 1. Ingredientes e composição das dietas experimentais para as fases inicial (um a 28 dias), crescimento ( 28 a 56 dias) e final (56 a 84 dias) para aves de corte criadas sob semiconfinamento

\begin{tabular}{|c|c|c|c|}
\hline Ingrediente (\%) & Inicial & Crescimento & Final \\
\hline Milho & 62,131 & 63,952 & 72,013 \\
\hline Farelo de soja $45 \%$ & 32,406 & 30,652 & 22,641 \\
\hline Óleo de soja & 0,645 & 1,518 & 1,674 \\
\hline Sal comum & 0,431 & 0,330 & 0,255 \\
\hline Suplemento mineral ${ }^{(1)}$ & 0,100 & 0,100 & 0,100 \\
\hline Suplemento vitamínico ${ }^{(2)}$ & 0,100 & 0,100 & 0,100 \\
\hline DL- metionina (99\%) & 0,148 & 0,168 & 0,110 \\
\hline L-lisina $\mathrm{HCl}(78 \%)$ & 0,109 & 0,116 & 0,048 \\
\hline Cloreto de colina $70 \%$ & 0,070 & 0,050 & 0,050 \\
\hline Fosfato bicálcico & 2,059 & 1,323 & 1,068 \\
\hline Calcário calcítico & 0,000 & 0,191 & 0,033 \\
\hline Inerte $^{(3)}$ & 1,801 & 1,500 & 1,908 \\
\hline Total & 100,00 & 100,00 & 100,00 \\
\hline \multicolumn{4}{|l|}{ Composição calculada } \\
\hline Proteína bruta (\%) & 20,00 & 19,373 & 16,316 \\
\hline Energia metabolizável (kcal/kg) & 2900 & 3.000 & 3.100 \\
\hline Cálcio (\%) & 0,600 & 0,490 & 0,350 \\
\hline Fósforo total (\%) & 0,702 & 0,561 & 0,490 \\
\hline Fósforo disponível (\%) & 0,489 & 0,351 & 0,298 \\
\hline Sódio (\%) & 0,190 & 0,150 & 0,120 \\
\hline Metionina + cistina digestível (\%) & 0,710 & 0,716 & 0,597 \\
\hline Lisina digestível (\%) & 1,041 & 1,006 & 0,766 \\
\hline Treonina digestível (\%) & 0,676 & 0,654 & 0,550 \\
\hline Arginina digestível (\%) & 1,261 & 1,211 & 0,984 \\
\hline Triptofano digestível (\%) & 0,219 & 0,210 & 0,170 \\
\hline Valina digestível (\%) & 0,843 & 0,815 & 0,689 \\
\hline
\end{tabular}

${ }^{(1)}$ Composição= Mn: 75mg; Fe: 50mg; Zn: 70mg; Cu: 8,50mg; Co: 2mg; I: 1,5mg; Se: 0,12mg; veículo q.s.p. $1.000 \mathrm{~g}$.

${ }^{(2)}$ Composição= vit.A:12.000UI; vit. $\mathrm{D}_{3}$ : 2200UI; vit.E: 30UI; vit. $\mathrm{B}_{1}$ : 2,2mg; vit. $\mathrm{B}_{2}$ : $6 \mathrm{mg}$; vit. $\mathrm{B}_{6}$ : 3,3mg; vit. $\mathrm{B}_{12}$ : 16mg; niacina: 2,500mg; ácido pantotênico: $13 \mathrm{mg}$; vit. $\mathrm{K}_{3}$ : 2,5mg; ácido fólico: $1 \mathrm{mg}$; antioxidante: $10 \mathrm{mg}$ : veículo q.s.p. $1.000 \mathrm{~g}$.

${ }^{(3)}$ Areia lavada.

As medidas da resistência à quebra óssea foram realizadas nas tíbias direitas, in natura, previamente descarnadas, utilizando-se uma máquina universal de ensaios mecânicos (EMIC ${ }^{\circledR}$ - modelo DL100 KW). Registrou-se a força aplicada no momento anterior à ruptura do osso e os valores foram expressos em quilograma força por milímetro $(\mathrm{kgf} / \mathrm{mm})$. Para as análises de cálcio, fósforo e cinzas, foram utilizadas as tíbias esquerdas, identificadas com placas de alumínio, mergulhadas em éter de petróleo por 48 horas e, posteriormente, secas em estufa de ventilação 
forçada a $60^{\circ} \mathrm{C}$ por 48 horas. Na sequência, as tíbias foram moídas em moinho de bola. As análises de cálcio, fósforo e cinzas da tíbia foram realizadas conforme Silva e Queiroz (2002) e expressas na base da matéria seca desengordurada.

Os dados foram submetidos às análises de variância e, posteriormente, à analise de regressão. As estimativas dos níveis ótimos de cálcio foram feitas por meio dos modelos linear response plateau (LRP) e polinomial quadrático. As análises estatísticas foram realizadas utilizando-se o programa SAS 9.1 (2009).

\section{RESULTADOS E DISCUSSÃO}

Na fase inicial de um a 28 dias de idade, o nível de $\mathrm{Ca}$ influenciou $(\mathrm{P} \leq 0,05)$ o consumo da dieta, o teor de cálcio na tíbia e a resistência à quebra óssea. Entre os sexos, verificou-se que não houve influência para conversão alimentar e teores de fósforo, de cálcio e de cinzas na tíbia. Não houve efeito da interação $(\mathrm{P}>0,05)$ nível de Ca da dieta versus sexo para todas as variáveis estudadas (Tab. 2).

De acordo com os resultados para o consumo da dieta (CD), os modelos LRP e quadrático ajustaram-se, estimando-se os níveis de 0,95 e $1,07 \%$ de $\mathrm{Ca}$, conforme as equações $\mathrm{CD}=$ 1251,90 - 187,20 (0,95-Ca); $\mathrm{P}=0,08$, e $\mathrm{CD}=$ $886,89+685,16 \mathrm{Ca}-318,74 \mathrm{Ca}^{2} ; \mathrm{P}=0,07$, respectivamente. Alves et al. (2002), ao estudarem a inclusão de Ca nas dietas de frangos de corte de um a 21 dias, suplementados em duas fontes, verificaram efeito significativo quando utilizaram o carbonato de cálcio e estimaram o nível de 0,67\% de Ca para o CD.

Tabela 2. Consumo de dieta (CD), ganho de peso (GP), conversão alimentar (CA), fósforo na tíbia (PT), cálcio na tíbia $(\mathrm{CaT})$, cinzas na tíbia $(\mathrm{CT})$ e resistência à quebra óssea $(\mathrm{RQO})$ de machos $(\mathrm{M})$ e fêmeas $(\mathrm{F})$ da linhagem ISA Label, alimentadas com dietas que continham quatro níveis de Ca no período de um a 28 dias de idade

\begin{tabular}{|c|c|c|c|c|c|c|c|c|c|c|}
\hline \multirow{2}{*}{ Variável } & \multicolumn{3}{|c|}{ Probabilidade } & \multirow{2}{*}{ Sexo } & \multicolumn{4}{|c|}{ Nível de cálcio (\%) } & \multirow{2}{*}{ Média } & \multirow{2}{*}{$\begin{array}{l}\text { CV } \\
(\%)\end{array}$} \\
\hline & $\mathrm{Ca}$ & Sexo & Ca*sexo & & 0,60 & 0,82 & 1,04 & 1,26 & & \\
\hline \multirow{3}{*}{ CD (g/ave) } & \multirow{3}{*}{0,0382} & \multirow{3}{*}{0,0010} & \multirow{3}{*}{0,3024} & M & 1210,13 & 1240,16 & 1303,33 & 1300,00 & 1263,41 & \multirow{3}{*}{3,42} \\
\hline & & & & $\mathrm{F}$ & 1161,34 & 1213,67 & 1221,07 & 1183,33 & 1194,85 & \\
\hline & & & & Média & 1185,74 & 1226,91 & 1262,20 & 1241,67 & & \\
\hline \multirow{3}{*}{ GP (g/ave) } & \multirow{3}{*}{0,1001} & \multirow{3}{*}{0,0098} & \multirow{3}{*}{0,7931} & M & 453,11 & 497,62 & 510,53 & 474,93 & 484,05 & \multirow{3}{*}{6,21} \\
\hline & & & & $\mathrm{F}$ & 435,84 & 459,07 & 459,88 & 442,80 & 448,40 & \\
\hline & & & & Média & 444,48 & 478,34 & 485,21 & 458,87 & & \\
\hline \multirow{3}{*}{ CA (g/g) } & \multirow{3}{*}{0,2317} & \multirow{3}{*}{0,3763} & \multirow{3}{*}{0,4409} & M & 2,677 & 2,498 & 2,558 & 2,739 & 2,617 & \multirow{3}{*}{4,57} \\
\hline & & & & $\mathrm{F}$ & 2,671 & 2,644 & 2,659 & 2,677 & 2,663 & \\
\hline & & & & Média & 2,674 & 2,571 & 2,608 & 2,708 & & \\
\hline \multirow{3}{*}{ PT (\%) } & \multirow{3}{*}{0,4379} & \multirow{3}{*}{0,5096} & \multirow{3}{*}{0,5011} & M & 9,29 & 9,05 & 9,44 & 9,37 & 9,29 & \multirow{3}{*}{4,42} \\
\hline & & & & $\mathrm{F}$ & 8,78 & 9,27 & 9,29 & 9,36 & 9,17 & \\
\hline & & & & Média & 9,03 & 9,16 & 9,36 & 9,37 & & \\
\hline \multirow{3}{*}{ CaT (\%) } & \multirow{3}{*}{0,0179} & \multirow{3}{*}{0,7116} & \multirow{3}{*}{0,3021} & M & 20,93 & 20,98 & 21,96 & 23,26 & 21,78 & \multirow{3}{*}{5,05} \\
\hline & & & & F & 20,41 & 22,33 & 21,04 & 22,68 & 21,61 & \\
\hline & & & & Média & 20,67 & 21,65 & 21,50 & 22,97 & & \\
\hline \multirow{3}{*}{ CT (\%) } & 0 & 08612 & 05309 & M & 48,03 & 46,19 & 48,91 & 48,92 & 48,01 & 420 \\
\hline & $0,3 / 10$ & 0,8612 & 0,3398 & $\mathrm{~F}$ & 47,05 & 48,24 & 47,99 & 49,35 & 48,16 & 4,20 \\
\hline & & & & Média & 47,54 & 47,22 & 48,46 & 49,13 & & \\
\hline RQO & & & & M & 9,46 & 10,58 & 11,76 & 11,61 & 10,85 & 1280 \\
\hline (kgf/mm) & 0,0448 & 0,0310 & 0,8419 & $\mathrm{~F}$ & 8,62 & 9,11 & 9,59 & 10,64 & 9,49 & 13,88 \\
\hline & & & & Média & 9,04 & 9,84 & 10,68 & 11,13 & & \\
\hline
\end{tabular}

CV = coeficiente de variação.

Conforme mencionado, os níveis de Ca da dieta influenciaram $(\mathrm{P}<0,05)$ a deposição de cálcio na tíbia (CaT), sendo os resultados ajustados pelos modelos linear e LRP. Pelo modelo LRP, estimou-se o nível 0,95\% de Ca na dieta, para otimizar a deposição óssea desse mineral, segundo a equação: $\mathrm{CaT}=22,235+4,468$ (Ca0,95). Em virtude das diferentes relações Ca:P 
devido à variação dos níveis de Ca das dietas, foi possível inferir que, nos níveis 0,60 e $0,82 \%$ de Ca, o status metabólico das aves caracterizou-se por hipocalcemia, que induz um estímulo à secreção do paratormônio e à síntese da vitamina D. Consequentemente, é possível que tenha ocorrido aumento na concentração de cálcio no plasma sanguíneo, devido à mobilização de cálcio ósseo, até que o nível máximo $(0,95 \%$ de Ca) fosse atingido, obtendo-se relação Ca:P mais estreita e próxima à recomendada para frangos de corte (Rath et al., 2000). Fato semelhante foi encontrado por Sá et al. (2004b) e Muniz et al. (2007), que trabalharam com a exigência de cálcio para frangos de corte na fase inicial e observaram aumento na deposição de CaT, com o aumento dos níveis de Ca das dietas, estimando os níveis de 0,93 e 0,75\% de Ca para maximizar o CaT, respectivamente.

Os resultados da resistência à quebra óssea (RQO) foram ajustados pelos modelos linear e LRP. Pelo LRP, foi estimado o nível de 1,16\% de Ca na dieta, segundo a equação $\mathrm{RQO}=11,127$ - 3,73 (1,16 - Ca) para a máxima RQO, ocorrendo a partir desse nível um platô na resposta. Sá et al. (2004b) estimaram o nível de 1,20\% de Ca na dieta para máxima RQO de frangos de corte de um a 21 dias de idade. Fanatico et al. (2005), ao avaliarem o desempenho de frangos de linhagem de crescimento lento e rápido, verificaram que aves de linhagem de crescimento rápido obtiveram maior RQO do que as de crescimento lento, o que se justifica pela sua necessidade em sustentar maior peso corporal.

Aves submetidas às dietas com baixos níveis de cálcio possuem habilidade em adaptarem-se eficientemente a essas dietas, devido ao aumento da capacidade absortiva e ao decréscimo na excreção do mineral, sendo esse processo mais pronunciado em aves em crescimento (Alves et al., 2002; Bar et al., 2003; Driver et al., 2005; Yan et al., 2005). A alta eficiência de absorção do cálcio pelas aves submetidas às dietas deficientes, na fase inicial de vida, pode explicar os resultados encontrados neste trabalho para a maioria das variáveis avaliadas, uma vez que não foram observados efeitos significativos dentro do intervalo estudado, até mesmo nos níveis de 0,60 e $0,82 \%$, muito abaixo daqueles recomendados na literatura para frangos de corte. Este resultado está de acordo com os encontrados por Driver et al. (2005).

No período de 28 a 56 dias de idade, observou-se efeito $(\mathrm{R}-0,05)$ do níve $l$ de $\mathrm{Ca}$ para todas as variáveis ósseas avaliadas, no entanto, para as variáveis de desempenho, isso não ocorreu. Constatou-se que todas as variáveis foram influenciadas pelo sexo $(\mathrm{P}<0,01)$, com exceção dos teores de fósforo, cálcio e cinzas na tíbia. Não houve efeito de interação $(\mathrm{P}>0,05)$ nível de Ca da dieta versus sexo para nenhuma variável, exceto para a resistência à quebra óssea, o que demonstra que machos e fêmeas apresentaram comportamento diferenciado para essa variável (Tab. 3).

Com relação à deposição de fósforo na tíbia (PT), foi possível ajustar os modelos linear e LRP. Pelo LRP, a interseção da reta ascendente com o platô correspondeu ao nível de 1,01\% de $\mathrm{Ca}$, ponto a partir do qual as respostas de PT das aves se estabilizaram $(\mathrm{PT}=8,550-1,084(1,01-$ Ca)).

Quanto à deposição de cálcio na tíbia (CaT), o ajuste foi possível pelo modelo linear $\mathrm{CaT}=$ $17,961+1,717$ Ca. Sá et al. (2004a), ao trabalharem com frangos de corte de 22 a 42 dias, encontraram a máxima deposição de CaT com o nível de 1,01\% de Ca na dieta.

Ao avaliar os resultados do teor de cinzas na tíbia (CT) das aves, constatou-se que somente o modelo linear ajustou-se adequadamente: CT= 38,816 + 6,031 Ca. Ao trabalharem com frangos de corte e avaliarem o efeito na qualidade óssea, no turnover ósseo e no metabolismo de cálcio e fósforo, Williams et al. (2000) relataram que não ficou clara a evidência de que o baixo conteúdo de cinzas nos ossos possa ser devido a problemas nutricionais. Segundo os autores, fatores genéticos ou crescimento rápido de algumas linhagens em função da máxima mineralização óssea, podem ser os responsáveis pelo baixo teor de cinzas nos ossos. 
Tabela 3. Consumo de dieta (CD), ganho de peso (GP), conversão alimentar (CA), fósforo na tíbia (PT), cálcio na tíbia (CaT), cinzas na tíbia (CT) e resistência à quebra óssea (RQO) de machos $(\mathrm{M})$ e fêmeas (F) da linhagem ISA Label, alimentadas com dietas que continham quatro níveis de Ca no período de 28 a 56 dias de idade

\begin{tabular}{|c|c|c|c|c|c|c|c|c|c|c|}
\hline \multirow{2}{*}{ Variável } & \multicolumn{3}{|c|}{ Probabilidade } & \multirow[b]{2}{*}{ Sexo } & \multicolumn{4}{|c|}{ Nível de cálcio (\%) } & \multirow{2}{*}{ Média } & \multirow{2}{*}{$\begin{array}{c}\text { CV } \\
\%\end{array}$} \\
\hline & $\mathrm{Ca}$ & Sexo & Ca*sexo & & 0,49 & 0,68 & 0,87 & 1,06 & & \\
\hline \multirow{3}{*}{ CD (g/ave) } & \multirow{3}{*}{0,2448} & \multirow{3}{*}{0,0001} & \multirow{3}{*}{0,9232} & $\mathrm{M}$ & 2773,74 & 2909,45 & 2907,19 & 2851,45 & 2860,46 & \multirow{3}{*}{4,56} \\
\hline & & & & $\mathrm{F}$ & 2407,48 & 2475,04 & 2566,19 & 2468,13 & 2479,21 & \\
\hline & & & & Média & 2590,61 & 2692,25 & 2736,69 & 2659,79 & & \\
\hline \multirow{3}{*}{ GP (g/ave) } & \multirow{3}{*}{0,3395} & \multirow{3}{*}{0,0001} & \multirow{3}{*}{0,6966} & M & 1134,86 & 1186,52 & 1194,95 & 1188,18 & 1176,13 & \multirow{3}{*}{ 3,95 } \\
\hline & & & & $\mathrm{F}$ & 918,74 & 916,85 & 948,06 & 926,20 & 927,46 & \\
\hline & & & & Média & 1026,80 & 1051,69 & 1071,51 & 1057,19 & & \\
\hline \multirow{3}{*}{ CA (g/g) } & \multirow{3}{*}{0,9043} & \multirow{3}{*}{0,0002} & \multirow{3}{*}{0,9179} & M & 2,445 & 2,452 & 2,435 & 2,406 & 2,434 & \multirow{3}{*}{4,57} \\
\hline & & & & $\mathrm{F}$ & 2,619 & 2,702 & 2,649 & 2,666 & 2,659 & \\
\hline & & & & Média & 2,532 & 2,577 & 2,542 & 2,536 & & \\
\hline \multirow{3}{*}{ PT (\%) } & \multirow{3}{*}{0,0093} & \multirow{3}{*}{0,3863} & \multirow{3}{*}{0,6648} & M & 7,91 & 8,33 & 8,42 & 8,65 & 8,33 & \multirow{3}{*}{3,14} \\
\hline & & & & $\mathrm{F}$ & 8,03 & 8,13 & 8,33 & 8,44 & 8,23 & \\
\hline & & & & Média & 7,97 & 8,23 & 8,38 & 8,55 & & \\
\hline \multirow{3}{*}{ СaT (\%) } & \multirow{3}{*}{0,034} & \multirow{3}{*}{0,8392} & \multirow{3}{*}{0,5285} & M & 18,73 & 19,90 & 18,82 & 19,84 & 19,32 & \multirow{3}{*}{4,17} \\
\hline & & & & $\mathrm{F}$ & 18,69 & 19,03 & 19,30 & 20,02 & 19,26 & \\
\hline & & & & Média & 18,71 & 19,46 & 19,06 & 19,93 & & \\
\hline \multirow{3}{*}{ CT (\%) } & 00042 & 02124 & 05995 & M & 41,19 & 44,56 & 44,03 & 45,76 & 43,88 & 3.42 \\
\hline & & $0, \angle 124$ & 0,5995 & $\mathrm{F}$ & 41,58 & 42,69 & 43,58 & 44,54 & 43,09 & 3,42 \\
\hline & & & & Média & 41,38 & 43,63 & 43,80 & 45,15 & & \\
\hline $\mathrm{B} \cap \cap$ (kof/mm) & & & & $\mathrm{M}$ & 15,52 & 18,91 & 18,69 & 22,65 & 18,94 & 970 \\
\hline 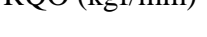 & 0,0002 & 0,0009 & 0,0338 & $\mathrm{~F}$ & 12,95 & 15,66 & 19,20 & 16,66 & 16,12 & (0) \\
\hline & & & & Média & 14,24 & 17,29 & 18,95 & 19,65 & & \\
\hline
\end{tabular}

CV = coeficiente de variação.

O desdobramento da interação nível de Ca versus sexo indicou que, para a resistência à quebra óssea (RQO), os modelos linear e LRP tiveram bom ajuste aos resultados dos machos, tendo sido estimado o nível de 0,779\% de Ca: RQO= 20,670 17,832 - (0,779 - Ca), pela equação do LRP. Com relação às fêmeas, os modelos linear, LRP e quadrático ajustaram-se adequadamente. A estimativa da exigência de Ca pelo modelo LRP forneceu o nível de 0,839\%: RQO=17,93 - 14,283 $(0,839-\mathrm{Ca})$, no entanto o modelo quadrático estimou 0,881\% de Ca na dieta: $\mathrm{RQO}=-10,093+$ $64,169 \mathrm{Ca}-36,422 \mathrm{Ca}^{2}$.

Na fase final de 56 a 84 dias, os resultados obtidos pela análise de variância, indicaram que nenhuma das variáveis foi influenciada $(\mathrm{P}>0,05)$ pelos níveis de Ca da dieta. No entanto, verificou-se que todas as variáveis foram influenciadas $(\mathrm{P}<0,01)$ pelo sexo. Houve efeito da interação do nível de Ca da dieta versus sexo somente para o ganho de peso (Tab. 4), caracterizando uma dependência entre os fatores estudados sobre essa variável, sendo, portanto, necessário analisá-la individualmente.

Em virtude da interação nível de Ca da dieta versus sexo para o ganho de peso (GP), fez-se o desdobramento e observou-se que os modelos LRP e quadrático ajustaram-se aos resultados dos machos. A estimativa fornecida pelo modelo LRP indicou o nível de 0,536\% de Ca: $\mathrm{GP}=1225,20$ 280,80 (0,536 - Ca); $\mathrm{P}=0,099$, porém o modelo quadrático estimou $0,686 \%$ de Ca para maximizar o GP: $\mathrm{GP}=952,36+842,47 \mathrm{Ca}-613,91 \mathrm{Ca}^{2} ; \mathrm{P}=$ 0,06 . Os resultados de GP das fêmeas foram ajustados pela equação linear decrescente, GP= 925,19 - 206,07 Ca, demonstrando que, à medida que os níveis de Ca das dietas aumentavam, ocorria redução no GP.

Essa redução pode ser devido à maior relação Ca: $\mathrm{P}$ (3,09: 1) no nível mais elevado (0,92\% de Ca). A relação Ca: $\mathrm{P}$ muito além de 2:1 reduz a biodisponibilidade do cálcio e do fósforo pela formação do complexo insolúvel de fosfato de cálcio nos intestinos das aves com adsorção de certos minerais traços, como $\mathrm{Mn}, \mathrm{Zn}, \mathrm{Cu}$ etc., prejudicando o desempenho delas (Alves et al., 2002; Rao et al., 2003; Schoulten et al., 2003). Este resultado está de acordo com os observados por Alves et al. (2002) e Bar et al. (2003), quando trabalharam com frangos de corte. 
Níveis nutricionais de cálcio...

Tabela 4. Consumo de dieta (CD), ganho de peso (GP), conversão alimentar (CA), fósforo na tíbia (PT), cálcio na tíbia (CaT), cinzas na tíbia (CT) e resistência à quebra óssea (RQO) de machos $(\mathrm{M})$ e fêmeas (F) da linhagem ISA Label, alimentadas com dietas que continham quatro níveis de Ca no período de 56 a 84 dias de idade

\begin{tabular}{|c|c|c|c|c|c|c|c|c|c|c|}
\hline \multirow{2}{*}{ Variável } & \multicolumn{3}{|c|}{ Probabilidade } & \multirow[b]{2}{*}{ Sexo } & \multicolumn{4}{|c|}{ Nível de cálcio (\%) } & \multirow{2}{*}{ Média } & \multirow{2}{*}{$\begin{array}{l}\text { CV } \\
(\%)\end{array}$} \\
\hline & $\mathrm{Ca}$ & Sexo & Ca*sexo & & 0,35 & 0,54 & 0,73 & 0,92 & & \\
\hline \multirow{3}{*}{ CD (g/ave) } & \multirow{3}{*}{0,2108} & \multirow{3}{*}{0,0001} & \multirow{3}{*}{0,2267} & $\mathrm{M}$ & 4143,67 & 4226,33 & 4283,45 & 4127,32 & 4195,19 & \multirow{3}{*}{2,57} \\
\hline & & & & $\mathrm{F}$ & 3190,00 & 3189,00 & 3094,00 & 3059,90 & 3133,23 & \\
\hline & & & & Média & 3666,83 & 3707,67 & 3688,72 & 3593,61 & & \\
\hline \multirow{3}{*}{ GP (g/ave) } & \multirow{3}{*}{0,1035} & \multirow{3}{*}{0,0001} & \multirow{3}{*}{0,0269} & M & 1172,83 & 1225,83 & 1242,65 & 1207,00 & 1212,08 & \multirow{3}{*}{4,15} \\
\hline & & & & $\mathrm{F}$ & 838,00 & 835,71 & 776,33 & 727,28 & 794,33 & \\
\hline & & & & Média & 1005,42 & 1030,77 & 1009,49 & 967,14 & & \\
\hline \multirow{3}{*}{ CA (g/g) } & \multirow{3}{*}{0,2871} & \multirow{3}{*}{0,0001} & \multirow{3}{*}{0,0726} & M & 3,535 & 3,448 & 3,446 & 3,421 & 3,463 & \multirow{3}{*}{4,47} \\
\hline & & & & F & 3,811 & 3,822 & 3,995 & 4,216 & 3,961 & \\
\hline & & & & Média & 3,673 & 3,635 & 3,721 & 3,819 & & \\
\hline \multirow{3}{*}{ PT (\%) } & \multirow{3}{*}{0,7364} & \multirow{3}{*}{0,0028} & \multirow{3}{*}{0,8527} & $\mathrm{M}$ & 7,44 & 7,50 & 7,68 & 7,71 & 7,58 & \multirow{3}{*}{5,47} \\
\hline & & & & $\mathrm{F}$ & 8,07 & 8,27 & 8,39 & 8,07 & 8,20 & \\
\hline & & & & Média & 7,75 & 7,89 & 8,03 & 7,89 & & \\
\hline \multirow{3}{*}{ CaT (\%) } & \multirow{3}{*}{0,1373} & \multirow{3}{*}{0,0067} & \multirow{3}{*}{0,3646} & M & 17,36 & 17,15 & 18,11 & 18,63 & 17,81 & \multirow{3}{*}{4,78} \\
\hline & & & & $\mathrm{F}$ & 18,30 & 18,92 & 19,75 & 18,74 & 18,93 & \\
\hline & & & & Média & 17,83 & 18,04 & 18,93 & 18,69 & & \\
\hline \multirow{3}{*}{ CT (\%) } & 07921 & 00004 & 05494 & $\mathrm{M}$ & 40,61 & 39,99 & 40,36 & 40,70 & 40,42 & $387>3$ \\
\hline & $0, / 921$ & 0,0004 & 0,5494 & $\mathrm{~F}$ & 42,01 & 43,98 & 43,88 & 43,55 & 43,35 & $3,8 /$ \\
\hline & & & & Média & 41,31 & 41,98 & 42,12 & 42,13 & & \\
\hline ROO (kgf/mm) & & & & M & 26,05 & 27,59 & 27,08 & 30,56 & 27,82 & $835>3$ \\
\hline 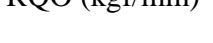 & 0,0569 & 0,0001 & 0,5263 & F & 19,13 & 21,94 & 20,49 & 21,47 & 20,75 & 8,35 \\
\hline & & & & Média & 22,59 & 24,76 & 23,78 & 26,01 & & \\
\hline
\end{tabular}

$\overline{\mathrm{CV}}$ = coeficiente de variação.

Skinner et al. (1992), ao trabalharem com frangos de corte de 42 a 56 dias de idade, não verificaram respostas sobre o desempenho quando forneceram dietas que continham 0,06 a $0,60 \%$ de Ca e 0,12 e/ou 0,24\% de fósforo disponível (Pd), e concluíram que a redução dos níveis de Ca e Pd para o referido período não apresentou efeitos adversos sobre o desempenho. Resultados semelhantes foram observados por Sá et al. (2004a) quando trabalharam com frangos de corte na fase de terminação (43 a 53 dias de idade).

Aves provenientes de linhagens de crescimento lento parecem ser menos sensíveis às variações dos níveis de cálcio nas dietas do que as de crescimento rápido, por manterem valores normais de cálcio no plasma até mesmo em níveis considerados altos (2\% de Ca) nas dietas (Hurwitz et al., 1995). Da mesma forma, Fanatico et al. (2008), ao avaliarem o desempenho de frangos de linhagem de crescimento lento e rápido, afirmaram que a genética dos frangos influenciou de forma marcante na incidência de discondroplasia tibial, isto é, as linhagens de crescimento rápido são mais propensas a problemas ósseos que as de crescimento lento.

\section{CONCLUSÕES}

Recomenda-se o nível de 1,16\% de Ca na dieta, baseado na máxima resistência à quebra óssea, de aves da linhagem ISA Label no período de um a 28 dias de idade, para ambos os sexos. Para o período de 28 a 56 dias de idade, os níveis de 0,78 e $0,88 \%$ de Ca na dieta foram recomendados em função da resistência à quebra óssea de machos e fêmeas, respectivamente, e para a fase final, de 56 a 84 dias de idade, recomenda-se o nível $0,69 \%$ de $\mathrm{Ca}$, considerando-se o ganho de peso das aves, independentemente do sexo.

\section{REFERÊNCIAS BIBLIOGRÁFICAS}

ALVES, E.L.; TEIXEIRA, A.S.; BERTECHINI, A.G. et al. Efeito dos níveis de cálcio em duas fontes sobre o desempenho de frangos de corte. Cienc. Agrotecnol., v.26, p.1305-1312, 2002. 
BAR, A.; SHINDER, D.; YOSEFI, S. et al. Metabolism and requirements for calcium and phosphorus in the fast-growing chicken as affected by age. Br. J. Nutr., v.89, p.51-60, 2003.

DRIVER, J.P.; PESTI, G.M.; BAKALLI, R.I. et al. Calcium requirements of the modern broiler chicken as influenced by dietary protein and age. Poult. Sci., v.84, p.1629-1639, 2005.

FANATICO, A.C.; PILLAI, P.B.; HESTER, P.Y. et al. Performance, livability, and carcass yield of slow- and fast-growing chicken genotypes fed low-nutrient or standard diets and raised indoors or with outdoor access. Poult. Sci., v.87, p.1012-1021, 2008.

FANATICO, A.C.; PILLAI, P.B.; CAVITT, L.C. et al. Evaluation of slower-growing broiler genotypes grown with and without outdoor access: growth performance and carcass yield. Poult. Sci., v.84, p.1321-1327, 2005.

HURWITZ, S.; PLAVNIK, I.; SHAPIRO, A. Calcium metabolism and requirements of chickens are affected by growth. J. Nutr., v.2, p.2679-2686, 1995.

MENDONÇA, M.O.; SAKOMURA, N.K.; SANTOS, F.R. et al. Níveis de energia metabolizável para machos de corte de crescimento lento em semiconfinamento. Rev. Bras. Zootec., v.37, p.1433-1440, 2008.

MUNIZ, E.B.; ARRUDA, A.M.V.; FASSANI, E.J. et al. Redução do nível de cálcio dietético para frangos de corte na fase inicial de crescimento. Rev. Caatinga, v.20, p.58-69, 2007.

NASCIMENTO, D.C.N.; SAKOMURA, N.K.; SIQUEIRA, J.C. et al. Exigências de lisina digestível para aves de corte da linhagem ISA Label criadas em semiconfinamento. Arq. Bras. Med. Vet. Zootec., v.61, p.1128-1138, 2009.

NASCIMENTO, D.C.N.; SAKOMURA, N.K.; SIQUEIRA, J.C. et al. Exigências de metionina + cistina digestível para aves de corte da linhagem ISA Label criadas em semiconfinamento. Rev. Bras. Zootec., v.38, p.869-878, 2009.

PINHEIRO, S.R.F. Níveis de fósforo, de cálcio e de cloreto de sódio para aves de linhagem de crescimento lento criadas em sistema semiconfinado. 2009. 104f. Tese (Doutorado) Faculdade de Ciências Agrárias e Veterinárias, Universidade Estadual Paulista, Jaboticabal, SP.
RAO, S.V.R.; PANDA, A.K.; RAJU, M.V.L.N. et al. Requirement of calcium for commercial broilers and white leghorn layers at low dietary phosphorus levels. Anim. Feed Sci. Technol., v.106, p.199-208, 2003.

RATH, N.C.; HUFF, G.R.; HUFF, W.E. et al. Factores regulating bone maturity and strength in poultry. Poult. Sci., v.79, p.1024-1032, 2000.

ROSTAGNO, H.S.; ALBINO, L.F.T.; DONZELE, J.L. et al. Tabelas brasileiras para aves e suínos: composição de alimentos e exigências nutricionais. Viçosa: UFV, 2005. $186 \mathrm{p}$.

SÁ, L.M.; GOMES, P.C.; ROSTAGNO, H.S. et al. Exigências nutricionais de cálcio para frangos de corte, nas fases de crescimento e terminação. Rev. Bras. Zootec., v.33, p.397-406, 2004a.

SÁ, L.M.; GOMES, P.C.; ALBINO, L.F.T. et al. Exigência nutricional de cálcio e sua biodisponibilidade em alguns alimentos para frangos de corte, no período de 1 a 21 dias de idade. Rev. Bras. Zootec., v.33, p.157-168, 2004b.

SCHOULTEN, N.A.; TEIXEIRA, A.S.; FREITAS, R.T.F. et al. Níveis de cálcio em rações de frangos de corte na fase inicial suplementadas com fitase. Rev. Bras. Zootec., v.32, p.1190-1197, 2003.

SILVA, D.J.; QUEIROZ, A.C. Análise de alimentos: métodos químicos e biológicos. 3.ed. Viçosa: UFV, 2002. 165p.

SKINNER, J.T.; ADAMS, M.H.; WATKINS, S.E. et al. Effect of calcium and nonphytate phosphorus levels fed during 42 to 56 days of age on performance and bone strength of male broilers. J. Appl. Poult. Res., v.1, p.167-171, 1992.

UNDERWOOD, E.J.; SUTTLE, N. F. Calcium. In: $\quad$ The mineral nutrition of livestock. 3.ed. Wallingford: Cabi Publishing, 1999. cap.4, p.67-104.

WILLIAMS, B.; WADDINGTON, D.; SOLOMON, S. et al. Dietary effects on bone quality and turnover, and $\mathrm{Ca}$ and $\mathrm{P}$ metabolism in chickens. Res. Vet. Sci., v.69, p.81-87, 2000.

YAN, F.; ANGEL, R.; ASHWELL, C. et al. Evaluation of the broiler's to adapt to an early moderate deficiency of phosphorus and calcium. Poult. Sci., v.84, p.1232-1241, 2005. 